\title{
Immunocytochemical features of obstructed saphenous vein coronary artery bypass grafts
}

\author{
J I BRODY, ${ }^{*} \dagger$ NANCY J PICKERING, ${ }^{*} \dagger$ G B FINK $\ddagger$ \\ From the Departments of Medicine, ${ }^{*}$ the Medical College of Pennsylvania and $\dagger$ Jefferson Park Hospital, and the \\ $\ddagger$ Department of Laboratory Medicine, Jefferson Park Hospital, Philadelphia, United States of America
}

SUMMARY The peroxidase-immunoperoxidase immunocytochemical method was used on 27 saphenous vein coronary artery bypass grafts, which had been resected because of recurrent angina, to identify in situ cellular and humoral elements possibly associated with graft occlusion. Immunostaining was performed on paraffin wax embedded control saphenous vein and graft sections incubated directly with primary antibodies against von Willebrand antigen (vWFAg), fibronectin, fibrinogen, leucocyte common antigen (LCA), lysozyme, vimentin, desmin, platelet factor 4, and thrombospondin. Antigens were visualised by a chromogen providing an orange-red immunoprecipitate at the site of epitope localisation.

The intraluminal, amorphous exudate present in most grafts was not composed simply of fibrin or fibrinogen, as previously thought, but was a multiprotein complex including wWFAg, fibronectin, thrombospondin and platelet factor 4 . Along with macrophages, these components probably enter the graft after haemodynamic, physical, and chemical injury to, and disruption of, the endothelial cell. Progressive myointimal proliferation and fibrosis of these grafts may be local repetitive responses to macrophages and platelets, cells previously known to participate in vascular disease.

The purpose of this investigation was to identify specific cellular and humoral constituents within surgically removed saphenous vein and coronary artery bypass grafts which may be associated with late graft obstruction.' Prior studies of these vessels by routine morphological assessment and staining may not have determined all the elements involved in reducing graft patency. ${ }^{2}$ In this study, therefore, we used the peroxidase-immunoperoxidase immunocytochemical technique ${ }^{3}$ to delineate previously unrecognised graft components which might have been associated with varying degrees of vasocclusion.

\section{Material and methods}

The grafts for study were obtained fresh from 16 consecutive patients who had further surgery because of unstable angina with angiographically documented luminal changes considered to be of clinical importance. Twenty seven grafts were removed from 14 men and two women, ranging in age from 35 to 72 years,

Accepted for publication 12 January 1989 with a mean of 58 years. Time elapsing since first bypass surgery varied from four to 13 years, with a mean of $9 \cdot 1$ years. Saphenous veins for identical analysis, used as controls, came from a separate group of 13 consecutive patients ( 11 men and two women, with a mean age of 60 years) about to receive their first coronary artery bypass.

All graft specimens were fixed in buffered formalin, embedded in paraffin wax, and sectioned at $5 \mathrm{mu}$. Tissue blocks from patients undergoing graft revision contained transverse sections of all the biopsy specimens from the site or sites of graft obstruction. Unfortunately, the sections were not necessarily identified by the vessel of origin, if more than one graft was involved, nor was the location of the single transection from each graft noted. Control sections were made by cutting $5 \mathrm{~mm}$ slices from each end and the centre of an unused portion of each venous segment and having the tissues processed as outlined above.

All biopsy specimens and control sections were stained first with Masson's trichrome, primarily to view the overall appearance of these vessels. The features being assessed were: the thickness of the vessel wall; whether separate wall layers were identifiable; 
the relative diameter of the lumen and its shape; and if the lumen was clear or contained an exudate.

Immunostaining of entire tissue sections mounted on slides was performed by the triple sandwich peroxidase-immunoperoxidase $\operatorname{method},{ }^{4}$ using aminoethyl carbazole as the chromogen which produced an easily seen red end-product, precipitating at the site of the antigen to be identified. The polyclonal and monoclonal primary antibodies were chosen particularly to identify graft constituents of potential pathogenic importance. The following primary polyclonal antibodies, directed against the specified antigen were made in rabbits: von Willebrand antigen (vWFAg), the immunoreactive portion of the factor VIII molecule necessary for platelet adhesion; fibronogen; lysozyme, a monocyte marker (all obtained from Ortho Diagnostic Systems, Raritan, New Jersey); thrombospondin, important as an adhesive protein, graciously provided by Dr Adam Asch, Cornell University Medical College, New York; and platelet factor 4 , a platelet marker released from $\alpha$ granules during platelet activation (American Diagnostica, Greenwich, Connecticut). Antibody to fibronectin, a protein functional in cell to cell interaction and adhesion, was purchased from BioGenex Laboratories, Dublin, California. The following were murine primary monoclones: leucocyte common antigen (LCA), which recognises all leucocytes; vimentin, which shows the presence of epitopes on endothelium, macrophages, and fibroblasts; and desmin, which shows smooth muscle (all purchased from BioGenex Laboratories, Dublin, California). All antibodies were used in dilutions to provide optimal staining without background non-specificity. Method controls consisted of assays without the primary antibody and a saline substitute, with completely irrelevant primary antibodies, and with chromogen alone.

\section{Results}

Fig 1 shows a control saphenous vein stained with Masson's trichrome before implantation. The vein had the normal structure ${ }^{56}$ of a comparatively thin intima and a proportionally thicker media and an outer adventitia. The endothelium was smooth, the lumen ellipsoid, sometimes circular, and clear. The three cuts taken from each venous segment were structurally similar to one another. Some veins, however, showed variable degrees of subendothelial thickening. When the control vein was immunostained the intima was found to be acellular, the media contained mainly smooth muscle marked by antidesmin with interspersed fibrocytes stained with antivimentin. Only vWFAg and fibronectin appeared as thin immunoprecipitates, sometimes limited to the inner circumference of the vessel, and at times also directly visible within the flat, single-layered endo-

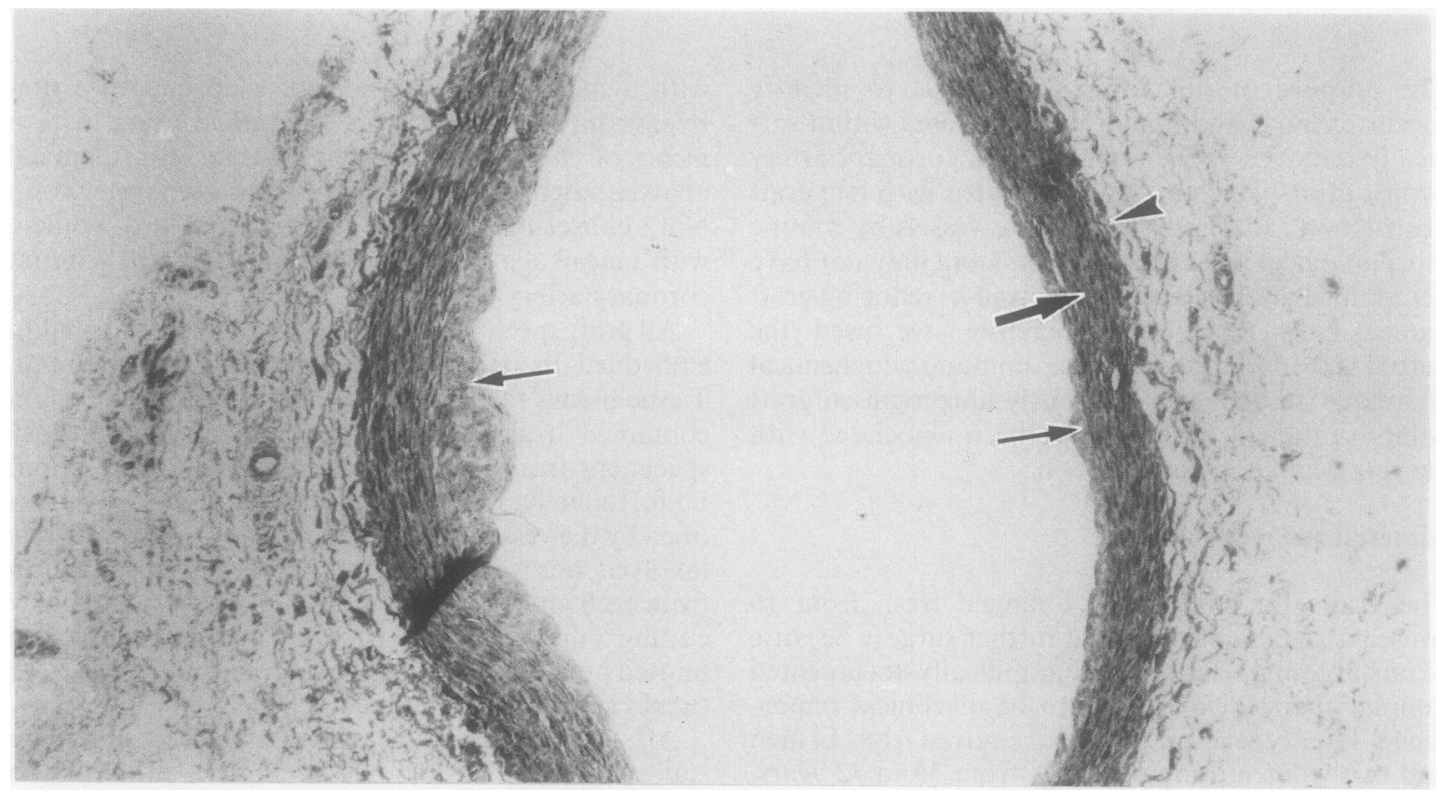

Fig 1 Preoperative control saphenous vein. Note relatively narrow intima (small arrows), thicker media (heavy arrow), and the outer, fibrillar adventitia (arrow head). The lumen is relatively symmetrical and clear. The different coats may vary somewhat in width in different sectors. 
thelial cells themselves (fig 2). Staining did not penetrate into the subendothelium. Thrombospondin, fibrinogen, and platelet factor 4 were not detected in the control veins.

Several types of changes were observed in the occluded grafts. The most common (11 of 27) was intimal myoproliferation of the wall. The lumen was reduced in calibre with a luminal exudate composed of an amorphous matrix and a cellular infiltrate. Higher magnification and immunostaining identified the cellular constituents of the exudate as mainly monocytes and macrophages reacting with anti-LCA and antilysozyme (fig 3). A few scattered cells with round nuclei, and a high nuclear:cytoplasmic ratio, also reacting with anti-LCA, had the appearance of lymphocytes.

The acellular portion of this exudate comprised four major proteins deposited as an extensive network within the obstructing graft lumen. Figs $4 a-c$ identify immunostained vWFAg, fibronectin, and thrombospondin, respectively, providing a scaffold on to which the mononuclear cells have clustered. The presence of platelets was confirmed by released platelet factor 4

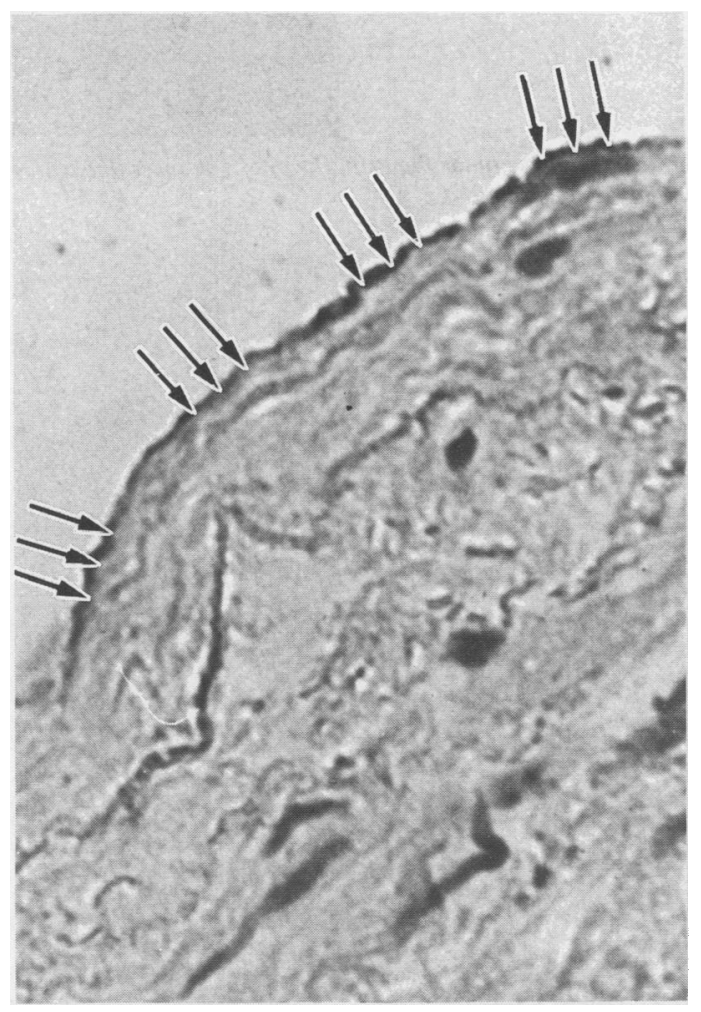

Fig 2 Arrows show $v W F A g$ as a linear immunoprecipitate along the inner surface of the control vein. Fibronectin had a similar appearance. (fig 4d). The variation in staining intensity may have reflected the different concentrations of these proteins in situ. Surprisingly, fibrinogen did not immunostain in any graft areas. The inability to locate this protein may have been related to circulatory wash-out, epitope degradation, and proteolysis, ${ }^{7}$ or to limited accessibility of the antibody to the antigen within the other proteins.

The predominant characteristics (fig 5) of the second most commonly observed graft abnormality (eight of 27) were an exudate-free, reduced calibre irregular lumen in which were seen large, undulating projections of a thickened graft wall. These may be larger, more extensive versions of the myointimal protrusions seen in the first graft type. Demarcations of the vessel wall layers were unclear. When stained with vimentin and desmin antibodies, this exuberant mural proliferation was shown to be composed of smooth muscle and fibroblasts.

The remaining grafts were multivariate in appearance. Some were a composite between the first two presentations-namely, increasing myointimal proliferation with a comparatively reduced intraluminal exudate. In other instances the vessel wall was devoid of smooth muscle and had become completely fibrotic. Finally, other grafts retained only a minuscle lumen with closure by mural fibrosis in which neovascularisation had occurred. Obvious red thrombi were not observed.

Different forms of graft obstruction were sometimes observed simultaneously, but separately, in the biopsy specimens taken from different grafts in the same patient. These changes did not seem to have occurred over related time. ${ }^{8}$ Had multiple, serial sections of individual grafts been available, however, they may have shown transitional gradations from one type of appearance to another.

\section{Discussion}

A major new observation of this study is that the intraluminal, acellular exudate previously thought to be simply fibrinogen or fibrin' is actually a complex multiprotein network. The in situ components shown by immunocytochemistry probably enter the graft after haemodynamic, physical, and chemical injury to the endothelium. These repetitive adverse stimuli include hypertension, hyperlipidemia, excessive shear stress, trauma at the perianastomotic graft site or exposure of the venous segment to an anomalous environment with an unusually high and pulsatile blood flow..$^{10-12}$

The finding of von Willebrand antigen, fibronectin, and thrombospondin, all products of endothelial synthesis, in the same place, and the deposition of wWFAg and fibronectin as aggregates, rather than in 


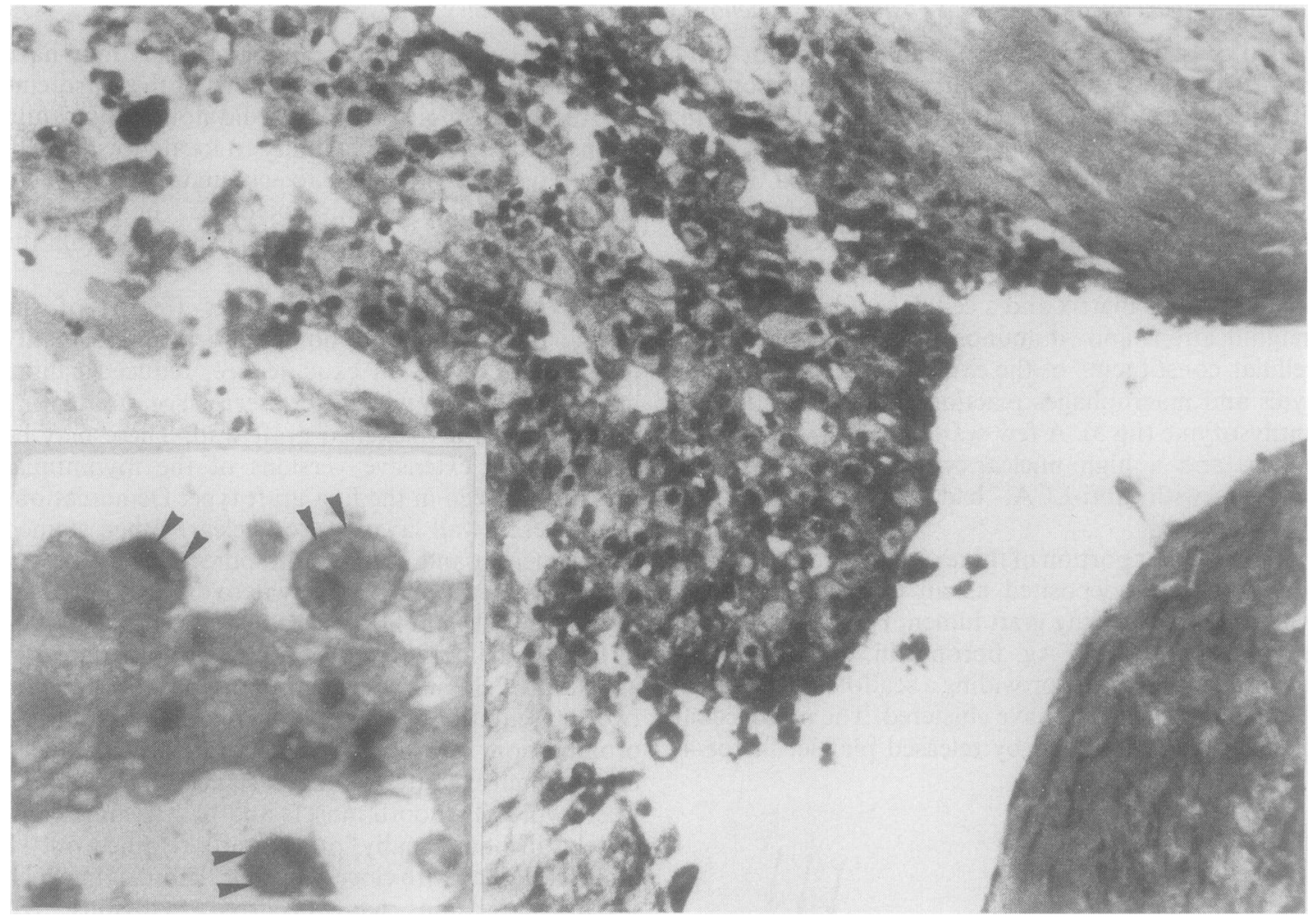

Fig 3 A photomicrograph to show macrophages as the major cell embedded in the amorphous meshwork. The inset illustrates a fine peripheral immunoprecipitate (arrowheads) formed on the macrophage with anti-LCA.

(a)

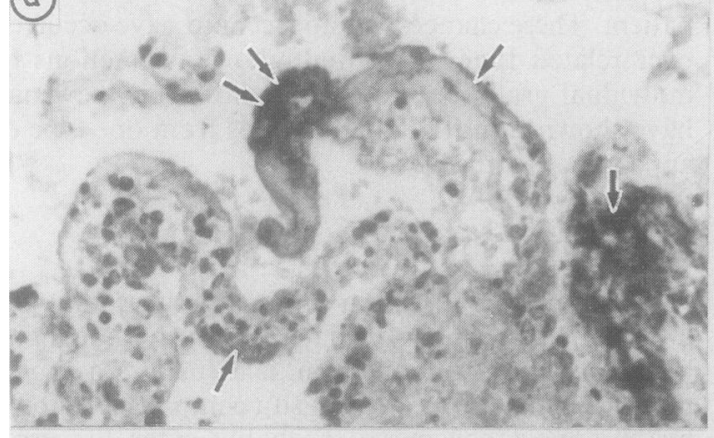

(b)

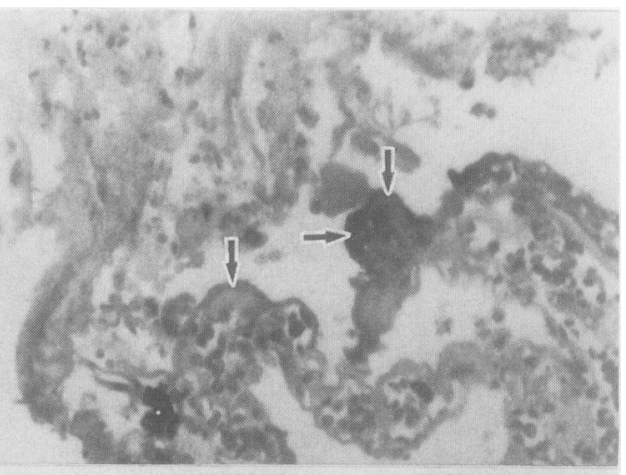

(c)

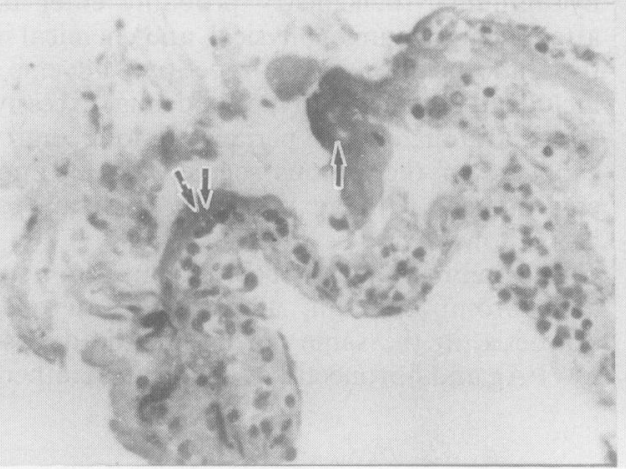

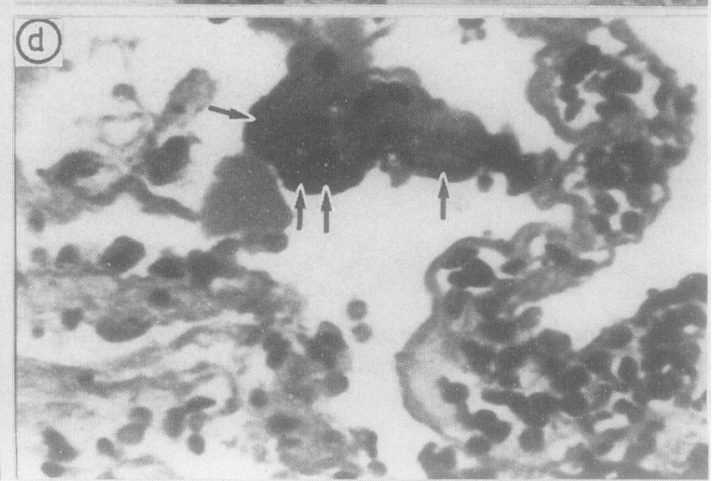

Fig 4 Four photographs of a single area immunostained with (a), anti-vWFAg; (b), anti-fibronectin; ( $c$ ), anti-

thromboplastin; and (d), anti-platelet factor $4.5 \mathrm{~d}$ has been enlarged to emphasise that platelet factor 4 has been deposited in very close opposition to fibronectin. Note, too, the difference in appearance of $v W F A g$ and fibronectin in this occlusive matrix from the fine immunoprecipitate in the control vein (fig 2) formed by the same antigens. 


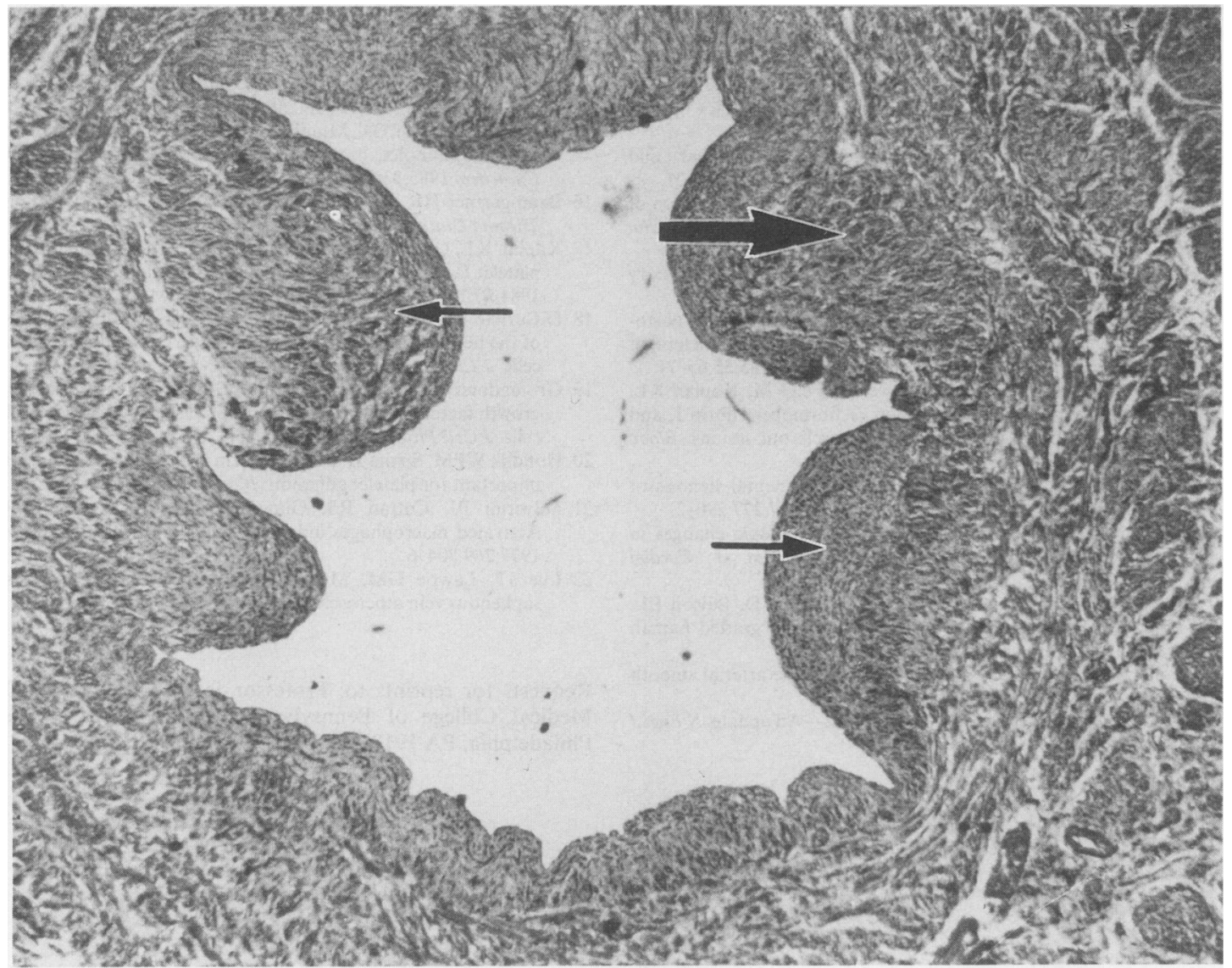

Fig 5 The second most common form of an occluded graft with a thickened intima-subendothelium (small arrows) into which extends a proliferative media (large arrow), ultimately leading to wall thickening, loss of layer demarcation, and reduced patency.

linear form, attest to endothelial cell disruption. ${ }^{13-15}$ It must be borne in mind that platelets are an additional source of these three proteins. Furthermore, the codistribution of platelet factor 4 , a platelet specific protein, within the intraluminal glycoprotein matrix confirms the presence of platelets and degranulation at the site of injury. ${ }^{16}{ }^{17}$ Finally, the migration of macrophages into the extracellular adhesive network is evidence of a chemotactic response to endothelial damage. ${ }^{1819}$

The different morphological and immunocytochemical presentations of these grafts imply that reduction in luminal patency may not be based on a single source or pathogenic sequence. The relative frequency, however, with which the glycoadhesive proteins vWFAg, fibronectin and thrombospondin were deposited within a large proportion of these grafts, coupled with their inherent physiological func- tion, suggest that they may be of importance in the genesis of graft obstruction. They may do this not only by interfering with blood flow themselves but also by providing an extracellular matrix into which monocytes, macrophages, and platelets migrate. These cells are known to have a role in vascular disease ${ }^{122021}$ and they may function similarly in this instance,$^{22}$ possibly as a source of mitogenic and proliferation-inducing biokines. Such macromolecules may stimulate replication of smooth muscle and fibroblasts with migration of these cells into the intima, resulting in luminal narrowing and graft failure.

Able technical assistance was provided by $\mathrm{Ms}$ Elizabeth Ross and Ms Marion B Brody. The manuscript was typed and collated by Ms Marene Urbany. 


\section{References}

1 Spray TH, Roberts WC. Changes in saphenous veins used as aortocoronary bypass grafts. Am Heart J 1977;94:500-16.

2 Hutchins GM. Pathological changes in aortocoronary bypass grafts. Ann Rev Med 1980;31:289-301.

3 Taylor CR. Immunoperoxidase technique. Practical and theoretical aspects. Arch Pathol Lab Med 1978;102:113-21.

4 Brody JI, Pickering NJ, Fink GB. Coronary artery deposition of factor VIII-related antigen in ischemic heart disease. Am J Clin Pathol 1986;86:269-73.

5 Smith SH, Geer JC. Morphology of saphenous vein-coronary bypass grafts. Am Heart J 1977;94:500-16.

6 Waller BF, Roberts WC. Remnant saphenous veins after aortocoronary bypass grafting: Analysis of 3394 centimeters of unused vein from 402 patients. Am J Cardiol 1985;55:65-71.

7 Bini A, Fenoglio J Jr, Sobel J, Owen J, Fejgl M, Kaplan KL. Immunochemical characterisation of fibrinogen, fibrin $\mathrm{I}$, and fibrin II in human thrombi and atherosclerotic lesions. Blood 1987;69:1038-45.

8 McNamara JJ, Darling RC, Linton RR. Segmental stenosis of saphenous vein autografts. $N$ Engl $J$ Med 1967;277:290-2.

9 Unni KK, Kottke BA, Titus JL, et al. Pathologic changes in aortocoronary saphenous vein grafts. Am J Cardiol 1974;34:526-32.

10 Brown BG, Cukinguan R, Peterson RB, Pierce D, Balson EL, Dodge HT. Perianstomatic arteriosclerosis in grafted human coronary arteries. Am J Cardiol 1982;49:968.

11 Ross RA, Glomset JA. Atherosclerosis and the arterial smooth muscle cells. Science 1973;180:1332-9.

12 Ross R. The pathogenesis of atherosclerosis-An update. $N$ Engl J Med 1986;314:488-500.
13 Jaffe EA, Mosher DF. Synthesis of fibronectin by cultured human endothelial cells. J Exp Med 1978;148:1779-91.

14 Rand JH, Sussman II, Gordon RE, Chu SV, Solomon V. Localisation of factor VIII-related antigen in human vascular subendothelium. Blood 1980;55:752-6.

15 Wright TN, Raugi GJ, Mumby SM, Bornstein P. Light microscopic immunolocalisation of thrombospondin. $J$ Histochem Cytochem 1985;33:295-302.

16 Baumgartner HR. Platelet interactions with vascular structures. Thromb Diath Haem 1972;(Suppl 51):161-76.

17 Kaplan KL, Owen J. Plasma levels of beta thromboglobulin and platelet factor 4 as indices of platelet activation in vivo. Blood 1981;57:199-202.

18 DiCorleto PE, de la Matte CA. Characterization of the adhesion of the human monocyte cell line U-932 to cultured endothelial cells. J Clin Invest 1985;75:153-61.

19 Grotendorst GR, Chang D, Griffin GL, et al. Platelet-derived growth factor is a chemoattractant for vascular smooth muscle cells. J Cell Physiol 1982;113:261-6.

20 Hondijk WPM, Sixma JJ. Fibronectin in artery subendothelium is important for platelet adhesion. Blood 1985;65:598-604.

21 Polverini PJ, Cotran RS, Gimbione MA Jr., Unanue ER. Activated macrophages induce vascular proliferation. Nature 1977;269:804-6.

22 Lue JT, Lawrie GM, Morris GC Jr. Aortocoronary bypass saphenous vein atherosclerosis. Am J Cardiol 1977;40:90614.

Requests for reprints to: Professor Jerome I Brody, MD, Medical College of Pennsylvania, 3300 Henry Avenue, Philadelphia, PA 19129, 215-842-6982, USA. 